\title{
Os lugares de atuação sacerdotal e as alusões ao Dia da Expiação em Hebreus
}

\author{
The places of priestly ministry and the Day \\ of Atonement allusions in the Epistle to the Hebrews
}

\section{Los lugares de actividad sacerdotal y las alusiones al Día de la Expiación en Hebreos}

\author{
Isaac Malheiros*
}

\begin{abstract}
RESUMO
O objetivo desta pesquisa é verificar onde o autor de Hebreus se refere ao sumo sacerdote no Lugar Santíssimo do santuário, e se essa referência está sempre vinculada ao Dia da Expiação no argumento do Autor. A pesquisa será feita através de uma análise linguística e exegética de palavras e expressões relacionadas à localização espacial da atuação sacerdotal em Hebreus. Essa análise utilizará como parâmetro a Septuaginta (LXX) e textos representativos do período do Segundo Templo, bem como a literatura judaica e cristã dos séculos próximos ao século I. Com base nesse artigo, é possível concluir que nem sempre o sumo sacerdote está vinculado ao Lugar Santíssimo e ao Dia da Expiação.

Palavras-chave: Hebreus. Sacerdócio. Dia da Expiação.
\end{abstract}

\begin{abstract}
The purpose of this research is to verify where the author of Hebrews refers to the high priest in the Most Holy Place of the sanctuary and its link to the Day of Atonement in the Author's argument. This research consists in a linguistic and exegetical analysis of words and phrases related to the location of the priesthood in Hebrews. This analysis used as a parameter the Septuagint (LXX) and representative texts of the Second Temple period, and also the Jewish and Christian literature from around the first century. Based on this article it is possible to conclude that the High Priest is not always linked to the Most Holy Place and to the Day of Atonement.

Keywords: Epistle to the Hebrews. Priesthood. Day of Atonement.

RESUMEN

El objetivo de esta investigación es verificar donde el autor de Hebreos se refiere al sumo sacerdote en el Lugar Santísimo del santuario, y si esa referencia está siempre vinculada al Día de la Expiación en el argumento del Autor. La investigación se hará a través de un análisis lingüístico y exegético de palabras y expresiones relacionadas con la localización espacial de la actuación sacerdotal en Hebreos. Este análisis utilizará como parámetro la Septuaginta (LXX) y textos representativos del período del Segundo Templo, así como la literatura judía y cristiana de los siglos próximos al siglo I. Basado en este artículo es posible concluir que el sumo sacerdote no está siempre vinculado al Lugar Santísimo y el Día de la Expiación. Palavras clave: Hebreos; Sacerdócio; Día de la Expiación.
\end{abstract}

* Mestre e doutorando em Teologia na Escola Superior de Teologia (EST, São Leopoldo-RS), membro do grupo de pesquisa Arqueologia e Religião (EST), bolsista da CAPES. E-mail: pr_isaac@yahoo.com 


\section{Introdução}

Parece razoável supor que sempre que Hebreus se refere ao sumo-sacerdote no Lugar Santíssimo, o Dia da Expiação está em mente, e tal suposição tem profundos desdobramentos na compreensão da teologia de Hebreus. O objetivo desta pesquisa é verificar onde o autor ${ }^{1}$ de Hebreus se refere ao sumo sacerdote no Lugar Santíssimo do santuário, e se essa referência está sempre vinculada ao Dia da Expiação no argumento do Autor. A pesquisa será feita através de uma análise linguística e exegética de palavras e expressões relacionadas à localização do sacerdócio em Hebreus. Essa análise utilizará como parâmetro a Septuaginta (LXX) (BRUCE, 1990, p. xlix) ${ }^{2}$ e textos representativos do período do Segundo Templo, como a literatura judaica pseudepígrafa, ${ }^{3}$ Fílon de Alexandria ${ }^{4}$ e Flávio Josefo. ${ }^{5}$ Também utilizará a literatura judaica e cristã dos séculos próximos ao século $\mathrm{I}^{6}{ }^{6}$

Esta pesquisa é necessária por que, além dos sacrifícios e rituais associados aos títulos sacerdote e sumo sacerdote, o lugar de atuação sacerdotal também tem sido utilizado para identificar qual é o pano de fundo conceitual do autor de Hebreus. Em poucas palavras, argumenta-se que, quando o Lugar Santíssimo é mencionado, então o Autor está pensando no Dia da Expiação. Vamos avaliar esse argumento.

\section{O sumo sacerdote em $\tau$ à ä $\gamma ı \alpha$}

Uma parte essencial dessa discussão gira em torno do sentido da expressão $\tau \grave{\alpha} \alpha ̋ \gamma 1 \alpha$ (adjetivo acusativo neutro plural de ö $\gamma 10 \varsigma$ ) em Hebreus. A

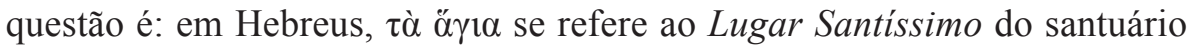
ou ao santuário como um todo? Uma comparação entre as traduções de

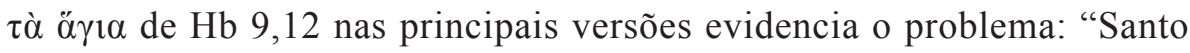
dos Santos" (ARA), "santo lugar" (AA), "santuário" (ARCF), "Lugar Santíssimo" (NVI), "santuário" (Reina Valera) e "holy place" (King James).

\footnotetext{
Diante da dificuldade de definir com precisão a autoria de Hebreus, nesta pesquisa o autor de Hebreus será identificado como "o Autor" ou "o autor de Hebreus".

2 Para pesquisas sobre a relação entre a LXX e Hebreus, ver GHEORGHITA, 2003; KISTEMAKER, 1961; STEYN, 2011.

3 Para analisar a literatura judaica pseudepígrafa, esta pesquisa utilizará como base a versão em inglês editada por CHARLESWORTH, 1983. E os textos gregos serão do texto eletrônico de EVANS, 2008. Ambos estão disponíveis em BIBLEWORKS, 2009.

4 Na análise dos textos de Fílon, esta pesquisa utilizará como base a versão editada por YONGE, 1854-1855. Disponível em BIBLEWORKS, 2009.

5 O texto grego de Flávio Josefo usado nesse artigo será o de NIESE, 1885-1895. Disponível em BIBLEWORKS, 2009.

${ }^{6} \mathrm{Na}$ análise de textos dos Pais da Igreja, esta pesquisa utilizará como base a versão editada por ROBERTS; DONALDSON; COXE, 1997. Os respectivos textos gregos estão disponíveis em BIBLEWORKS, 2009.
} 
Como registram os léxicos, a palavra grega ö $\gamma 10 \varsigma$ não se refere especificamente ao Lugar Santíssimo, mas ao santuário em geral.7 Em 1954, Henry S. Gehman concluiu que tò ö $\gamma 1 \alpha$, na LXX, se refere ao santuário

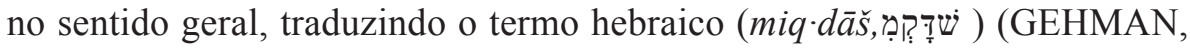
1954, p.345). Em 1989, Alwyn P. Salom (1989, p.221) concluiu o mesmo,

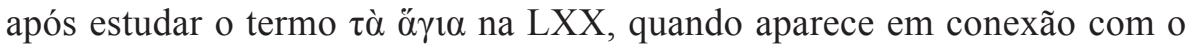
santuário, descobrindo que $\tau \grave{\alpha}$ ö $\gamma 1 \alpha$ quase sempre se refere ao santuário em geral. Salom concluiu que ő $\gamma 10 \varsigma$ aparece 170 vezes na LXX em conexão com o tabernáculo ou templo. Das 66 vezes que aparece no singular, 45 vezes referem-se ao santuário como um todo, 13 ao Lugar Santo, e 8 ao Lugar Santíssimo. Das 104 vezes que aparece no plural, 97 referem-se ao santuário como um todo, 6 ao Lugar Santo, e 1 ao Lugar Santíssimo. Como tà ă $\gamma 1 \alpha$ na LXX geralmente se refere ao santuário como um todo, pode-se argumentar que $\tau \grave{\alpha}$ ö $\gamma 1 \alpha$ tenha o mesmo sentido em Hebreus (SALOM, 1989, p. 219-227).

Mas, para entender o sentido de $\tau \grave{\alpha}$ ó $\gamma 1 \alpha$ em Hebreus do ponto de vista de sua audiência original, é importante examinar, também, o contexto mais amplo do uso contemporâneo de $\tau$ à ö $\gamma 1 \alpha$ durante o primeiro século. E foi o que Cosaert (2004, p. 91-103) fez em 2004, surpreendendo-se ao descobrir como esse tema havia sido largamente ignorado na academia. Ele examinou o uso de ärios nos livros pseudepígrafos, bem como nas obras de Fílon e Josefo. A pesquisa de Cosaert mostra que o uso judaico contemporâneo de $\tau \grave{\alpha}$ ö $\gamma 1 \alpha$ confirma as conclusões de Salom e Gehman: a forma plural $\tau \grave{\alpha} \alpha$ ó $\gamma 1 \alpha$ nunca é usada para descrever o Lugar Santíssimo sozinho, ela descreve exclusivamente todo o santuário em geral (COSAERT, 2004, p.102-103). Portanto, $\tau \grave{\alpha}$ ă $\gamma 1 \alpha$ não parece ter sido uma palavra costumeiramente usada no judaísmo do século I para se referir ao Lugar Santíssimo, e deve ser uma referência ao santuário como um todo em Hebreus.

\section{O sumo sacerdote "dentro do véu"}

Em Hb 6,19-20, o sumo sacerdote ( $\alpha \rho \chi \imath \rho \rho \varepsilon v ́ \varsigma)$ Jesus vai para "dentro

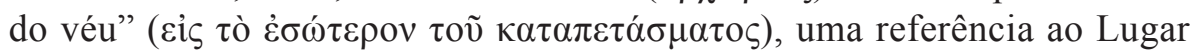
Santíssimo na LXX, onde a expressão "dentro do véu" aparece quatro vezes, e sempre como uma referência ao véu do Lugar Santíssimo (Êx 26,33; Lv 16,2, 12, 15; cf. Nm 18,7) (GANE, 2000, p.5-8). No entanto, há uma discussão a respeito de qual ritual do cerimonial levítico estaria no background dessa entrada no Santíssimo em Hb 6,19-20: o Dia da Expiação ou a Inauguração/Dedicação do santuário? ${ }^{8}$

\footnotetext{
Por exemplo, BAUER; DANKER; ARNDT; GINGRICH, 2000, p. 11.

Ver, por exemplo, o debate entre Norman Young e Richard Davidson em YOUNG, 2001, p. 165-173; YOUNG, 2002, p. 61-68; DAVIDSON, 2001, p. 175-190; DAVIDSON, 2002, p. 69-88.
} 
O debate se dá sobre a que véu do tabernáculo $\mathrm{Hb}$ 6,19-20 está se referindo. Mas a descrição de Jesus como sumo sacerdote (ả $\rho 1 \varepsilon \rho \varepsilon u ́ \varsigma)$ e o uso da expressão $\tau$ ò $\dot{\sigma} \sigma \omega ́ \tau \varepsilon \rho o v ~ \tau o v ̃ ~ \kappa \alpha \tau \alpha \pi \varepsilon \tau \alpha ́ \sigma \mu \alpha \tau o \varsigma$ na LXX sugerem que é o véu entre o Lugar Santo e o Santíssimo (ELLINGWORTH, 1993, p.347).

George Rice (1987, p.65-71) tentou desafiar essa interpretação, argumentando que esse véu poderia ser o primeiro véu, que dá acesso ao santuário como um todo. Posteriormente, Roy Gane respondeu, afirmando que, apesar da posição de Rice ser teoricamente possível, a LXX apoia fortemente a conclusão de que esse véu é o do Lugar Santíssimo (GANE, 2000, p.8).

Como já foi dito, a expressão "além do véu" ( $\pi \varepsilon \tau \alpha \dot{\alpha} \sigma \mu \alpha \tau \varsigma)$ aparece quatro vezes na LXX, e sempre como uma referência ao véu do Lugar Santíssimo (GANE, 2000, p.5-8). Portanto, aqui há uma forte evidência de que o Autor esteja falando da entrada no Lugar Santíssimo. No entanto, essa entrada no Santíssimo pode ser uma referência ao Dia da Expiação (Lv 16,1-3) ou à Inauguração/Dedicação do santuário (Ex 26,33-34; cf. 40,1-9; Lv 8,10-12; Nm 7,1; 2 Cr 5,7; 35,3). ${ }^{9}$

O propósito da entrada de Jesus "dentro do véu" não é explicado no contexto imediato de $\mathrm{Hb}$ 6,19-20. O Autor apenas menciona a entrada de Jesus "como precursor" e como sumo sacerdote, mas não explica ritualisticamente o porquê dessa entrada. Ele explicou anteriormente o propósito do sacerdócio de Jesus em favor dos que são tentados $(2,18 ; 4,15-16)$ e como tal sacerdócio fornece "expiação pelos pecados do povo" (1,3, 2,17, cf. $5,1)$. Porém, ele não desenvolve estes aspectos relacionando-os à entrada de Jesus além do véu em $\mathrm{Hb}$ 6,19-20.

Norman Young (2001, p.171) chama a atenção para o fato de que o único lugar do AT que fala sobre o sumo sacerdote indo "dentro do véu" é Levítico 16 (vv.2, 12, 15), que descreve o Dia da Expiação. Já a ideia de Moisés indo "dentro do véu" na Inauguração/Dedicação do santuário é apenas inferida, e não mencionada explicitamente em Êx 40, Lv 8 e Nm 7 , e esses textos jamais chamam Moisés de sumo sacerdote (YOUNG, 2002, p. 62). Portanto, argumenta-se que, em Hebreus, a Inauguração/Dedicação do santuário não estabeleceria uma relação tipológica entre Moisés e Jesus tão forte quanto a que usa o Dia da Expiação como pano de fundo.

No entanto, é preciso reconhecer que há, sim, em Hebreus, uma comparação explícita entre Moisés e Jesus. Em Hebreus, Moisés representa um tipo de Jesus (3,1-6; 9,15-24), atuando como um sumo sacerdote e

O tema da Inauguração/Dedicação do santuário é considerado relevante porque o livro de Hebreus se refere à inauguração do santuário celestial, em 9,21-23. 
rei, como inaugurador da aliança e do santuário, que entrou no santuário (inclusive no Santíssimo), como parte do ritual de inauguração (Êx 26,33; 40,1-9; Lv 8,10-12; Nm 7,1).

Embora não possuísse o título de sacerdote, foi Moisés quem inaugurou o santuário e a antiga aliança; e em Hebreus, Jesus, seu sangue e sua aliança também são comparados e contrastados com a atuação de Moisés na antiga aliança. E, no argumento de Hebreus, os sacrifícios relacionados com a inauguração da aliança (Hb 9,15-23) também têm função expiatória

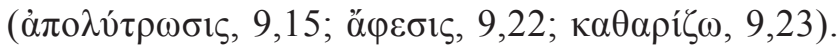

O fato de Moisés não ser literalmente um sacerdote não é um problema em Hebreus, pois Jesus também é sacerdote de outra linhagem, da ordem de Melquisedeque. $\mathrm{O}$ autor de Hebreus apresenta Jesus transitando nessas duas dimensões: como ministro do santuário (sacerdote e sumo-sacerdote) e mediador do concerto. Portanto, "dentro do véu" é uma referência à entrada no Lugar Santíssimo, mas não necessariamente ao Dia da Expiação.

\section{O sumo sacerdote "no céu"}

$\mathrm{Em} \mathrm{Hb} \mathrm{4,14,} \mathrm{o} \mathrm{grande} \mathrm{sumo} \mathrm{sacerdote} \mathrm{(} \alpha \rho \chi \varepsilon \rho \varepsilon \dot{\alpha} \alpha \mu \varepsilon \dot{\gamma} \gamma \alpha \nu)$ atravessou

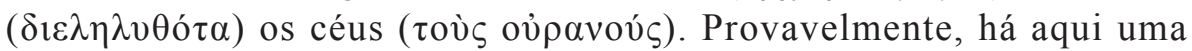
alusão ao conceito judaico de vários céus, em camadas, ${ }^{10}$ e a entrada do sumo sacerdote nos céus é uma metáfora tanto para a exaltação de Cristo quanto para o acesso a Deus.

Esse texto será analisado com mais profundidade posteriormente, mas é útil antecipar aqui uma discussão relacionada a ele. Uma boa parte dos comentaristas entende que a entrada de Cristo nos céus é representada pela entrada do sumo sacerdote no Santíssimo no Dia da Expiação, baseado no conceito do universo como um templo gigantesco, com o céu representando o Santíssimo deste grande templo cosmológico universal. Esse conceito de um santuário cosmológico é encontrado nos escritos de Josefo, Fílon de Alexandria, Clemente de Alexandria, e em grande parte da literatura gnóstica (MacRAE, 1984, p.177-180, 183-186).

Fílon ${ }^{11}$ e Josefo ${ }^{12}$ identificam o cosmos com o santuário, no qual o Lugar Santo e o Lugar Santíssimo representam a terra e o céu, respectivamente. Por

\footnotetext{
10 A literatura judaica descreve a existência de um céu (1 Enoque 14), três céus (Testamento de Levi 2-3; 2 Cor 12,2-4; 1 Enoque 71,1-5) e sete céus (Testamento de Levi 2-3; Apocalipse de Abraão 10,8; 19,4; 2 Enoque 3-22; 3 Baruque 11-17).

11 Legum Allegoriae iii.102; De Cherubim 23-26; De Plantatione 26-27, 50; Quis rerum divinarum Heres sit 75; De Congressu Eruditionisgratia 116-117; De Somniis i.185-187; De Vita Mosis ii.71-75, 88, 98, 102-103; De Specialibus Legibus i.66; Quaestiones et Solutiones in Exodum ii.52, 75, 82, 90-96.

12 Antiguidades, Livro III, 6:4.
} 
algum tempo, predominou na academia a ideia de que o pensamento platônico de Fílon de Alexandria poderia ser o principal pano de fundo conceitual de Hebreus. Nesse sentido, a ideia do santuário cosmológico prevaleceu, e a entrada no céu era vista como a entrada no Lugar Santíssimo. No entanto, em Hebreus 9,11, o "maior e mais perfeito tabernáculo" inteiro é identificado como algo "não feito por mãos, isto é, não desta criação", sem distinguir o Lugar Santíssimo e o Lugar Santo (ATTRIDGE, 1989, p.222-223), o que contradiz a interpretação cosmológica filônica.

Um problema adicional dessa interpretação é o fato de Hebreus não descrever o santuário celestial como o lugar de ideias eternas, mas como um lugar real, a sala do trono de Deus (COCKERILL, 2012, p.354). ${ }^{13}$ Para Fílon, o templo celestial que serviu de padrão para Moisés não era real, mas apenas um modelo incorpóreo. ${ }^{14}$ Esse tipo de santuário noético, incorpóreo, não pode conter rituais celestiais, não é uma realidade na qual alguém poderia entrar num santuário, tornar-se um sumo-sacerdote, oferecer sacrifício, inaugurar uma aliança, efetuar purificação e sentar-se em um trono, como em Hebreus (BUCHANAN, 1972. p. 134; HURST, 1990, p. 37; SCHENCK, 2007, p. 151 152, 166-167; BARNARD, 2012, p. 97-98). Um céu onde tais coisas podem acontecer está mais de acordo com a literatura judaica apocalíptica e mística do que com o conceito platônico do mundo das ideias.

Diante dessas evidências, muitos acadêmicos buscaram uma alternativa: "a linguagem ritualística de Hebreus é compatível com o modelo do judaísmo apocalíptico, em vez do modelo de Fílon” (JOHNSSON, 1989, p.108). Assim, tem ganhado força a ideia de que o santuário celestial de Hebreus reflete o conceito judaico de um santuário/templo no céu, que aparece no AT e na literatura apocalíptica judaica, e é mais facilmente justificável que a posição "santuário como cosmos", associada com o judaísmo helenístico platônico.

A estreita relação entre Hebreus, o AT e a literatura apocalíptica e mística judaica torna possível concluir que o autor de Hebreus reflete o conceito de um santuário celestial real (não metafórico ou platônico), no céu (não cosmológico), com dois compartimentos, onde já há atividade sacerdotal (diferentemente do modelo escatológico).

A interpretação de Hebreus que vê um santuário celeste com dois compartimentos (um Lugar Santo e o Santíssimo) geralmente recebe críticas por parte daqueles que alegam que não há um propósito para um Lugar Santo no santuário celestial, pois tal lugar seria um símbolo da falta de acesso à

\footnotetext{
13 Para outras diferenças entre o platonismo e Hebreus, ver WILLIAMSON, 970, p. 565-67; ADAMS, 2009. p. 133; ELLINGWORTH, 1993, p. 408.

14 De Vita Mosis ii.74-76.
} 
presença divina (Hb 9,8). Nessa concepção negativa, o Lugar Santo funciona como uma barreira para Deus, e é considerado incompatível com o santuário celeste e a mensagem de livre acesso a Deus (KOESTER, 1989, p. 158-159; SCHENCK, 2007, p. 147, 172; COCKERILL, 2012, p. 354-55). Tais intérpretes entendem, portanto, que o santuário celestial de Hebreus não contém dois compartimentos.

No entanto, a correspondência entre os santuários celestes e terrestres sugere que, assim como o tabernáculo terrestre tem dois compartimentos, o santuário celestial também possui dois compartimentos (MOFFITT, 2011, p.224). Apesar de Hebreus não dizer explicitamente que o tabernáculo celestial contém um Lugar Santo e um Santíssimo, está implícito um santuário de dois compartimentos no céu (MACKIE, 2007, p.165; ATTRIDGE, 1989, p.218, 223; MOFFITT, 2011, p.223-224; BARNARD, 2012, p.93).

Esse argumento é coerente com $\mathrm{Hb} 8,5$, que descreve o santuário ter-

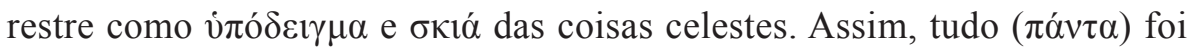
feito conforme o modelo ( $\tau \dot{\pi} \pi \mathrm{s}$ ) celestial. Essa correspondência vertical entre os santuários terrestres bicompartimentados e o celestial também é confirmada na literatura apocalíptica judaica (uma das influências conceituais de Hebreus), ${ }^{15}$ o que sugere que o santuário celestial de Hebreus tem dois compartimentos, como o tabernáculo terreno (RIBBENS, 2013, p.142).

O tema do acesso à presença de Deus em Hebreus não elimina a existência de uma cortina/véu no santuário celestial em Hebreus (6,19; 10,20), correspondente à cortina/véu do tabernáculo terreno (Hb 9,3), já que o santuário terrestre é cópia e sombra do celestial $(\mathrm{Hb} 8,5)$, onde essencialmente tudo correspondia ao modelo.

Hebreus não apresenta o fim de todas as barreiras de acesso a Deus, mas sim que essas barreiras não podem mais impedir o acesso do crente, demonstra como Cristo rompeu essas barreiras e abriu um caminho para que os crentes entrem na presença de Deus $(9,8 ; 10,18-19)$. Este acesso não significa que um véu/cortina não existe mais no santuário celestial, pois o acesso não é concedido a todas as pessoas, mas apenas aos crentes que foram limpos e aperfeiçoados $(10,14,19-20,22)$. Além disso, a existência dessas "barreiras" no céu, especialmente na sala do trono/Lugar Santíssimo celestial, mesmo num contexto de acesso a Deus, são muito comuns na literatura apocalíptica. ${ }^{16}$

Portanto, os destinatários de Hebreus muito provavelmente entendiam o santuário celeste como possuindo dois compartimentos, já que um santuário

\footnotetext{
153 Enoque 45,1; Testamento de Levi 3,4-9; Jubileus 31,14; 1Enoque 14,15-16, 20; 4Q405 Frag.19, 3-4; 4Q405 Frag. 15-16, 3.

16 2Enoque 3-22; Testamento de Levi 3,1-10; 3Baruque 11,1-14,2; 4Q405 Frag.15-16, 3; comparar com 2Co 12,2.
} 
de dois compartimentos era "um componente básico e imutável em todas as estruturas do santuário do antigo Israel” (BARNARD, 2012, p.110-111).

\section{O sumo sacerdote à direita de Deus e do seu trono}

Em Hebreus 8,1, o sumo sacerdote ( $\alpha \rho \chi 1 \varepsilon \rho \varepsilon ́ \alpha)$ está localizado "à destra

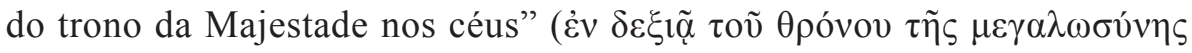

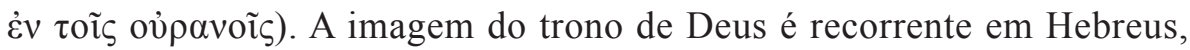
e indica exaltação $(1,8 ; 8,1 ; 12,2 ;$ cf. $1,3,13)$ ou acesso a Deus $(4,16)$. De fato, o trono de Deus também pode ser descrito como uma referência ao propiciatório do santuário, onde a glória de Deus se manifestava e diante do qual o sangue era aspergido no Dia da Expiação (Lv 16,14-15) (JOHNSSON, 1989, p.114).

No entanto, essa localização em $\mathrm{Hb} 8,1$ não tem essa conotação, pois o texto relaciona o sumo sacerdote ao santuário como um todo ( $\tau \tilde{\omega} v$ $\dot{\alpha} \gamma i ́ \omega v)$, e ao tabernáculo como um todo ( $\tau \tilde{\eta} \varsigma \sigma \kappa \eta v \tilde{\eta} \varsigma)$. Em Hb 10,11-12,

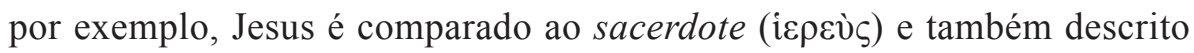

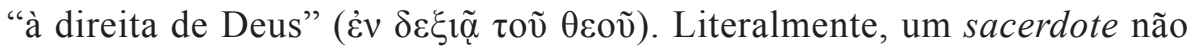
é a figura mais adequada para descrever a entrada no Santíssimo e a aproximação ao propiciatório, visto que no santuário terrestre o sacerdote não tinha esse direito. Ou seja, se em Hebreus a destra de Deus é lugar para o sacerdote e para o sumo sacerdote, isso indica que o Autor não tem o Dia da Expiação em mente.

Portanto, em vez de uma referência exclusiva ao propiciatório do santuário (e, consequentemente, ao Dia da Expiação), o "trono da Majestade" é uma expressão metafórica que indica a exaltação de Cristo.

\section{Considerações finais}

Em Hebreus, o sumo sacerdote nem sempre está vinculado ao Lugar

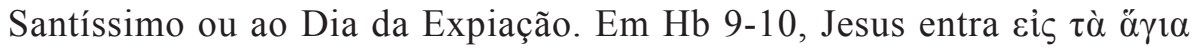
$(9,12$; cf. 9,24; 10,19), uma expressão que geralmente indica o santuário como um todo na LXX, e não o Lugar Santíssimo (SALOM, 1989, p. 219-227; GEHMAN, 1954, p. 337-348; COSAERT, 2004, p. 91-103). Essa

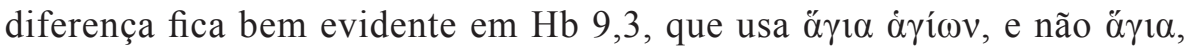
para se referir especificamente ao Lugar Santíssimo (ELLINGWORTH, 1993, p.447). Assim, ver em $\tau$ ò ő $\gamma 1 \alpha$ uma referência ao Lugar Santíssimo implicaria um novo significado para o termo tò ó $\gamma 1 \alpha$, um significado que não era tão comum (ELLINGWORTH, 1993, p.447).

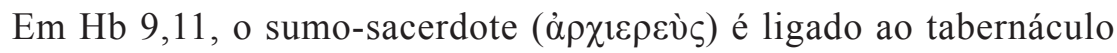

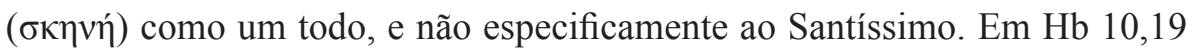


e 21, o "grande sacerdote" (i $\varepsilon \rho \varepsilon \dot{\alpha} \alpha \mu \varepsilon \dot{\gamma} \gamma \alpha v$ ) é ligado ao "santuário" ( $\tau \tilde{\omega} v$

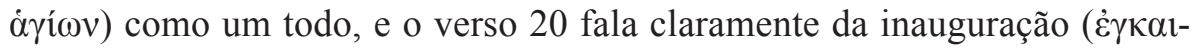

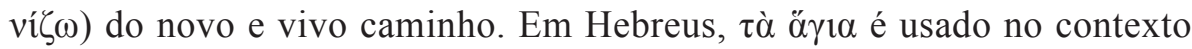
da obra do sumo-sacerdote, mas contrasta o que ele fazia em geral (e não só anualmente) com o que Cristo fez. Não é possível demonstrar que em cada ocorrência de $\tau \dot{\alpha}$ ö $\gamma ı \alpha$ a entrada do sumo sacerdote no contexto do Dia da Expiação está em vista.

À margem da discussão linguística, o principal argumento erudito em favor de $\tau \alpha$ ò $\alpha 1 \alpha$ como uma referência específica ao Lugar Santíssimo é a correspondência tipológica entre a entrada de Jesus no céu e a entrada do sumo sacerdote no Lugar Santíssimo no Dia da Expiação. É quase um consenso. ${ }^{17}$

No entanto, esse consenso parece repousar mais sobre a supervalorização de uma ideia popularizada do que sobre argumentos convincentes. Não há um vínculo linguístico entre Hebreus e Levíticos 16 nesse ponto. A LXX usa o singular tò ă $\gamma$ iov para se referir ao Lugar Santíssimo, e não o

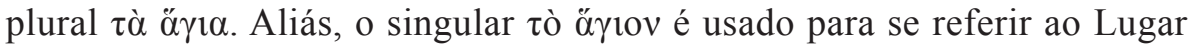
Santíssimo apenas em Levíticos 16, e em nenhum outro lugar do AT. ${ }^{18} \mathrm{O}$ uso do plural $\tau \alpha$ à $\gamma 1 \alpha$ afasta Hebreus de Levíticos 16 e o aproxima dos demais textos da LXX.

Cortez expõe o problema afirmando que "a visão de uma tipologia de Dia de Expiação para a ascensão de Jesus é tão aceita entre os estudiosos hoje que é difícil para outra ideia até mesmo ser mencionada" (CORTEZ, 2008, p.39). Há uma espécie de hegemonia de pensamento, uma posição apresentada como inquestionável unanimidade (YOUNG, 2001, p.166). Mas supervalorizar o Dia da Expiação como o tema principal e dominante em Hebreus não ajuda a explicar adequadamente a relação entre as alusões ao Dia da Expiação e os demais rituais aludidos explicitamente no texto, igualmente importantes, igualmente relacionados com o sacrifício, ascensão e sacerdócio de Jesus. É preciso reconhecer que o Autor mistura e integra alusões a vários rituais e eventos diferentes na exposição do sacrifício, da ascensão e do ministério celestial de Jesus.

Os pesquisadores de Hebreus deveriam analisar mais detidamente os textos que descrevem Jesus como sacerdote ou sumo sacerdote, tentando identificar neles todos os elementos que possam ser referência a algum ritual do cerimonial levítico.

Por exemplo, ATTRIDGE, 1989, p. 245; YOUNG, 2002, p. 61-68; ELLINGWORTH, 1993, p. 445448; WILSON, 1987, p. 149-153; SPICQ, 1952-1953, p. 257; MORRIS, 1983, p. 84; CODY, 1960, p. 170-202; LANE, 1991, p. cxxiv.

18 Lv 16,2, 3, 16, 17, 20, 23, 27. COSAERT, 2004, p. 98, nota 35. 


\section{Referências bibliográficas}

ADAMS, Edward. The cosmology of Hebrews. In: BAUCKHAM, Richard et alii (Eds.). The Epistle to the Hebrews and christian theology. Grand Rapids: Eerdmans, 2009.

ATTRIDGE, Harold W. The Epistle to the Hebrews. Hermeneia. Philadelphia: Fortress, 1989.

BARNARD, Jordy. The mysticism of Hebrews: exploring the role of jewish apocalyptic mysticism in the Epistle to the Hebrews. Tübingen: Mohr Siebeck, 2012.

BAUER, W.; DANKER, F. W.; ARNDT, W. F.; GINGRICH, F. W. Greek-English lexicon of the New Testament and other early christian literature. Chicago: The University of Chicago Press, 2000.

BIBLEWORKS. Bibleworks 8: Versão 8.0.013z.1. Norfolk: Bibleworks, LLC, 2009.

BRUCE, Frederick F. The Epistle to the Hebrews. Grand Rapids: Eerdmans, 1990.

BUCHANAN, George Wesley. To the Hebrews. The Anchor Bible, vol. 36. Garden City:

Doubleday, 1972.

CHARLESWORTH, James H. (ed.) The Old Testament Pseudepigrapha. 2 vols. Garden City: Doubleday, 1983.

COCKERILL, Gareth Lee. The Epistle to the Hebrews. New International Commentary on the New Testament. Grand Rapids: Eerdmans, 2012.

CODY, Aelred. Heavenly sanctuary and liturgy in the Epistle to the Hebrews: the achievement of salvation in the Epistle's perspective. St. Meinrad: Grail, 1960.

CORTEZ, Felix H. "The anchor of the soul that enters within the veil": the ascension of the "son" in the letter to the Hebrews. Tese (Doutorado). Berrien Springs: Andrews University, 2008.

COSAERT, Carl P. The use of örios for the sanctuary in the Old Testament Pseudepigrapha, Philo, and Josephus. Andrews University Seminary Studies, vol. 42, 2004.

DAVIDSON, Richard M. Christ's entry within the veil' in Hebrews 6:19-20: The Old Testament background. Andrews University Seminary Studies, vol. 39, p. 175-190, 2001.

DAVIDSON, Richard M. Inauguration or atonement? A response to Norman Young's "Old Testament background to Hebrews 6:19-20 revisited". Andrews University Seminary Studies, vol. 40, p. 69-88, 2002.

ELLINGWORTH, Paul. The Epistle to the Hebrews. New International Greek Testament Commentary. Grand Rapids: Eerdmans, 1993.

EVANS, Craig A. The Greek Pseudepigrapha. OakTree Software, 2008.

GANE, Roy E. Re-opening katapetasma ("veil") in Heb 6:19. Andrews University Seminary Studies, vol. 38, 2000.

GEHMAN, Henry S. 'A $\gamma$ เos· in the Septuagint, and Its Relation to the Hebrew Original. Vetus Testamentum. v. 4, p. 337-348, 1954. Disponível em: < http://booksandjournals.brillonline.com/content/journals/10.1163/156853354x00280>. Acesso em 15 out. 2016.

GHEORGHITA, Radu. The role of the Septuagint in Hebrews. Tübingen: Mohr Siebeck, 2003. HURST, Lincoln D. The Epistle to the Hebrews: its background of thought. Society for New Testament Studies Monograph Series 65. Cambridge: Cambridge University, 1990.

JOHNSSON, William G. Day of Atonement allusions. In: HOLBROOK, Frank B. (Ed.). Issues in the Book of Hebrews. Daniel and Revelation Committee Series, vol. 4. Silver Spring: Biblical Research Institute General Conference of Seventh-day Adventists, 1989. 
KISTEMAKER, Simon. The Psalm citations in the Epistle to the Hebrews. Amsterdam: Van Soest, 1961.

KOESTER, Craig R. The dwelling of God: the tabernacle in the Old Testament, intertestamental jewish literature, and the Old Testament. Catholic Biblical Quarterly Monograph Series 22. Washington, DC: Catholic Biblical Association of America, 1989.

LANE, William. Hebrews 1-8. Word Bible Commentary. N. 47a. Dallas: Word, 1991.

MACKIE, Scott D. Eschatology and exhortation in the Epistle to the Hebrews. WUNT 2/223.

Tübingen: Mohr Siebeck, 2007.

MacRAE, George. W. The temple as a house of revelation in the Nag Hammadi texts. In: MADSEN, Truman G. (ed). The Temple in Antiquity: Ancient Records and Modern Perspectives. Provo: Brigham Young University, 1984, p. 175-190. Disponível em: < https://rsc.byu. edu/archived/temple-antiquity-ancient-records-and-modern-perspectives/temple-house-revelation-nag>. Acesso em: 2 ago. 2016.

MOFFITT, David M. Atonement and the logic of resurrection in the Epistle to the Hebrews. Novum Testament Supplements, vol. 141. Leiden: Brill, 2011.

MORRIS, Leon. Hebrews. Bible Study Commentary. Grand Rapids: Zondervan, 1983.

RIBBENS, Benjamin J. Levitical sacrifice and heavenly cult in Hebrews. Tese (Doutorado). Wheaton: Wheaton College, 2013. Disponível em: <http://espace.wheaton.edu/lr/a-sc/archives/ theses/201307-PhD-BITH-RibbensBen.pdf>. Acesso em 12 jul. 2015.

RICE, George E. Hebrews 6:19: analysis of some assumptions concerning katapetasma. Andrews University Seminary Studies. vol. 25, p. 65-71, 1987.

ROBERTS, Alexander; DONALDSON, James; COXE, A. Cleveland. (Eds.). The ante-nicene fathers: translations of the writings of the fathers down to A.D. 325.8 vols. Oak Harbor: Logos Research Systems, 1997.

SALOM, Alwyn P. Ta Hagia in the Epistle to the Hebrews. In: HOLBROOK, Frank B. (Ed.). Issues in the Book of Hebrews. Daniel and Revelation Committee Series, n. 4. Silver Springs: Biblical Research Institute, General Conference of Seventh-day Adventists, 1989.

SCHENCK, Kenneth. Cosmology and eschatology in Hebrews: the settings of the sacrifice. Society for New Testament Studies Monograph Series 143. Cambridge: Cambridge University Press, 2007.

SPICQ, Ceslas. L'Épitre aux Hébreux. 2 vols. Paris: J. Gabalda et Cie. Éditeurs, 1952-1953.

STEYN, Gert. A quest for the assumed LXX vorlage of the explicit quotations in Hebrews. Göttingen: Vandenhoeck \& Ruprecht, 2011. Disponível em: <http://scholar.sun.ac.za/bitstream/10019.1/1121/1/Steyn,\%20GJ.pdf>. Acesso em 14 nov. 2015.

WILSON, R. McL. Hebrews. The New Century Bible Commentary. Grand Rapids: Eerdmans, 1987.

YOUNG, Norman H. The Day of Dedication or the Day of Atonement? The Old Testament background to Hebrews 6:19-20 revisited. Andrews University Seminary Studies. vol. 40, p. 61-68, 2002.

YOUNG, Norman H. Where Jesus has gone as a forerunner on our behalf' (Hebrews 6:20). Andrews University Seminary Studies. vol. 39, p. 165-173, 2001.

Submetido em: 21-11-2017

Aceito em: 19-12-2017 